\title{
The WSES/SICG/ACOI/SICUT/ACEMC/SIFIPAC guidelines for diagnosis and treatment of acute left colonic diverticulitis in the elderly
}

Paola Fugazzola ${ }^{*}\left(\mathbb{D}\right.$, Marco Ceresoli ${ }^{2}$, Federico Coccolini ${ }^{3}$, Francesco Gabrielli², Alessandro Puzziello ${ }^{4}$, Fabio Monzani ${ }^{5}$, Bruno Amato ${ }^{6}$, Gabriele Sganga7, Massimo Sartelli ${ }^{8}$, Francesco Menichetti ${ }^{9}$, Gabriele Adolfo Puglisi ${ }^{3}$, Dario Tartaglia ${ }^{3}$, Paolo Carcoforo ${ }^{10}$, Nicola Avenia ${ }^{11}$, Yoram Kluger $^{12}$, Ciro Paolillo ${ }^{13}$, Mauro Zago ${ }^{14}$, Ari Leppäniemi ${ }^{15}$, Matteo Tomasoni ${ }^{1}$, Lorenzo Cobianchi ${ }^{1}$, Francesca Dal Mas ${ }^{16}$, Mario Improta ${ }^{17}$, Ernest E. Moore ${ }^{18}$, Andrew B. Peitzman ${ }^{19}$, Michael Sugrue ${ }^{20}$, Vanni Agnoletti ${ }^{21}$, Gustavo P. Fraga ${ }^{22}$,

Dieter G. Weber ${ }^{23}$, Dimitrios Damaskos ${ }^{24}$, Fikri M. Abu-Zidan ${ }^{25}$, Imtiaz Wani ${ }^{26}$, Andrew W. Kirkpatrick ${ }^{27}$, Manos Pikoulis ${ }^{28}$, Nikolaos Pararas ${ }^{29}$, Edward Tan ${ }^{30}$, Richard Ten Broek ${ }^{30}$, Ronald V. Maier ${ }^{31}$, R. Justin Davies ${ }^{32}$, Jeffry Kashuk ${ }^{33}$, Vishal G. Shelat ${ }^{34}$, Alain Chicom Mefire ${ }^{35}$, Goran Augustin ${ }^{36}$, Stefano Magnone ${ }^{37}$, Elia Poiasina ${ }^{37}$, Belinda De Simone ${ }^{38}$, Massimo Chiarugi ${ }^{3}$, Walt Biff ${ }^{39}$, Gian Luca Baiocchi ${ }^{40}$, Fausto Catena ${ }^{41}$ and Luca Ansaloni ${ }^{1}$

\begin{abstract}
Acute left colonic diverticulitis (ALCD) in the elderly presents with unique epidemiological features when compared with younger patients. The clinical presentation is more nuanced in the elderly population, having higher in-hospital and postoperative mortality. Furthermore, geriatric comorbidities are a risk factor for complicated diverticulitis. Finally, elderly patients have a lower risk of recurrent episodes and, in case of recurrence, a lower probability of requiring urgent surgery than younger patients. The aim of the present work is to study age-related factors that may support a unique approach to the diagnosis and treatment of this problem in the elderly when compared with the WSES guidelines for the management of acute left-sided colonic diverticulitis. During the $1^{\circ}$ Pisa Workshop of Acute Care \& Trauma Surgery held in Pisa (Italy) in September 2019, with the collaboration of the World Society of Emergency Surgery (WSES), the Italian Society of Geriatric Surgery (SICG), the Italian Hospital Surgeons Association (ACOI), the Italian Emergency Surgery and Trauma Association (SICUT), the Academy of Emergency Medicine and Care (ACEMC) and the Italian Society of Surgical Pathophysiology (SIFIPAC), three panel members presented a number of statements developed for each of the four themes regarding the diagnosis and management of ALCD in older patients, formulated according to the GRADE approach, at a Consensus Conference where a panel of experts participated. The statements were subsequently debated, revised, and finally approved by the Consensus Conference attendees. The current paper is a summary report of the definitive guidelines statements on each of the following topics: diagnosis, management, surgical technique and antibiotic therapy.
\end{abstract}

Keywords: Acute diverticulitis, Elderly, Surgery in elderly

*Correspondence: paola.fugazzola@gmail.com

${ }^{1}$ IRCCS Policlinico San Matteo Foundation, General Surgery, Pavia, Italy

Full list of author information is available at the end of the article

\section{Background}

Diverticulitis results from a microscopic or macroscopic perforation of a diverticulum due to diverticular inflammation and focal necrosis. Diverticulitis can present in 
about $10-25 \%$ of patients with diverticulosis and can be uncomplicated (symptomatic uncomplicated diverticular disease, SUDD) and complicated. Complicated diverticulitis could be associated with the formation of abscess, fistula, bowel obstruction, or frank perforation. Patients of Western nations are overwhelmingly likely to have leftsided diverticulosis (90\% of cases), whereas those of Asian and African descent are likely to have the right-sided disease (70-74\% of cases) [1]. The prevalence of acute left colonic diverticulitis (ALCD) increases with age. The lifetime prevalence of diverticular disease, extracted from the Health Search Database of Italian General Practitioners, increases from $10 \%$ among patients under 50 years old to $33 \%$ among patients between 60 and 69 years old [2]. However, in the last decade, the prevalence of hospitalization for acute diverticulitis has increased among patients under 70 years old, while remaining unchanged for patients aged over 70 years [3].

ALCD in elderly patients has different epidemiological features when compared with younger patients. The clinical presentation is more nuanced in the elderly population. In an observational study by Lizardi-Cervera focusing on patients with ALCD [4], only $50 \%$ of patients older than 65 years presented with abdominal pain in the lower quadrants, $17 \%$ had a fever and $43 \%$ did not have leucocytosis. On the contrary, a higher proportion of older patients presented with diverticular bleeding [4, 5]. According to some authors [6-8], the risk factor for complicated acute diverticulitis is not older age by itself, but the presence of comorbidities, often associated with advanced age.

The results of two meta-analyses on patients with ALCD $[7,9]$ showed that the risk of urgent surgery during a primary episode is similar when comparing patients younger and older than 50 years of age. However, as can be expected, the in-hospital mortality of patients admitted for ALCD is higher among older patients; in the last decade, increased in-hospital mortality was demonstrated in the patient cohort aged 70 years and older [2]. Again, it is not clear whether the age itself or the associated comorbidities influence mortality. In a large study by Sirinthornpunya [6], the multivariate analysis showed comorbidities as the only significant risk factor for inhospital mortality.

Similar to elderly patients with severe sepsis who underwent alimentary tract surgery [10], advanced age persisted as an independent predictor of postoperative mortality after emergency surgery for ALCD. The observed mortality rates were $1.6 \%$ in patients younger than 65 years, $9.7 \%$ in patients between 65 and 79 years and $17.8 \%$ in patients above 80 years [11].
Regarding the risk of recurrence after the first episode of ALCD, according to most authors [7, 9, 12], the risk is significantly lower in older patients compared with younger ones. In a large study on patients over 67 years of age [12], the proportion of patients without recurrence was $83 \%$ and among patients over 80 years the recurrences were even less likely. The need for urgent surgery for recurrence was also significantly lower among older patients (cumulative risk $7.3 \%$ in patients younger than 50 years vs $4.3 \%$ in patients older than 50 years) [9]. Currently, there are no specific guidelines for the management of ALCD focusing on elderly patients.

The aim of the Consensus Conference held in Pisa in September 2019 was to investigate age-related factors that could influence a different approach, compared with the WSES guidelines for the management of acute leftsided colonic diverticulitis $[13,14]$, in terms of diagnosis and management of elderly patients with ALCD.

\section{Material and methods}

The World Society of Emergency Surgery (WSES) along with the Italian Society of Surgical Physiopathology (SIFIPAC), the Italian Society of Geriatric Surgery (SICG), the Italian Hospital Surgeons Association (ACOI), the Italian Emergency Surgery and Trauma Association (SICUT) and the Academy of Emergency Medicine and Care (AcEMC) nominated a scientific committee for the development of guidelines for the diagnosis and the treatment of ALCD in the elderly patients.

Similar to the age cut-off established in the guidelines on appendicitis and on cholecystitis in the elderly [15], we elected to define "elderly" as patients aged over 65 years.

Four areas of interest were identified by the scientific committee: diagnostic, management, surgical technique and antibiotic therapy. Using the PICO framework, many focus questions were generated and constructed for each area of interest. An electronic bibliography search on PubMed and EMBASE was used to perform a systematic review of the existing literature. Researchers reviewed the literature and used the GRADE methodology to develop evidence-based responses to the questions grading the quality of evidence and assigning the recommendation's strength [16]. According to GRADE methodology, the quality of evidence was assessed and classified, into four levels: high, moderate, low and very low; the consequent recommendations were made based on the level of evidence and were classified into two levels: strong recommendation in favour or against; weak recommendation (suggestion) in favour or against. 
During the $1^{\circ}$ Pisa Workshop of Acute Care \& Trauma Surgery in Pisa (Italy) in September 2019, each proposed statement was discussed, along with the results of the literature review, with the participation of a panel of experts, including members of WSES, SIFIPAC, SICG, ACOI, SICUT, AcEMC. Each statement was then voted upon by the audience and was approved if it reached at least $80 \%$ of votes in favour. Where there was discordance, the statement was improved with panel input until approval was granted by the assembly.

Diverticulitis severity was graded according to the WSES ALCD classification (Fig. 1) [17].

\section{Results}

Diagnosis

1. Could the diagnosis of acute left colonic diverticulitis be based on only clinical signs, symptoms and laboratory test in elderly patients?

Statement 1.1 In the elderly population, we suggest against basing the diagnosis of acute left colonic diverticulitis on only patient clinical signs, symptoms and laboratory tests. [Conditional recommendation, very low-quality evidence]

Statement 1.2 We suggest that elderly patients presenting with abdominal guarding or pain in the lower left abdomen on physical examination undergo appropriate imaging for suspected diverticulitis, regardless of the value of leukocytes and of C-reactive protein (CRP). [Conditional recommendation, very low-quality evidence]

As stated in the WSES Guidelines [13, 14] in the general population, the clinical diagnosis of ALCD alone is not sufficiently accurate for patients with suspected diverticulitis. However, pain in the lower left abdomen, elevate temperature and absence of vomiting could suggest a diagnosis of ALCD $[13,14]$. The reported sensitivity and specificity of the clinical diagnosis of ALCD in the general population are 0.68 and 0.98 , respectively [18].

Several authors have developed clinical scoring systems in order to minimize secondary care diagnostics in suspected ALCD [19-21], but these were validated on small sample sizes composed of the general population. Bolkenstein et al. [22] developed a diagnostic prediction model distinguishing complicated from uncomplicated ALCD, validated on 475 patients presenting to the emergency department (ED) with a clinically suspected episode of diverticulitis. This model included abdominal guarding, CRP level and leucocytes count. The final model would have avoided secondary care diagnostics in $25 \%$ of all patients, with a negative predictive value (NPV) of $96 \%$. Still, the mean age of the tested population was 61 years of age. Many studies have demonstrated that clinical manifestations are insufficient for the diagnosis of ALCD. Longstreth et al., in a retrospective electronic record-based analysis, reported that nearly $5 \%$ of patients with severe diverticulitis presented without fever or leucocytosis [23]. In the study by Kaser et al. on 247 patients [24], if a computed tomography $(\mathrm{CT})$ scan was performed only with a CRP above $50 \mathrm{mg} / \mathrm{l}, 46 \mathrm{CT}$ scan would have been avoided; however, 12 cases of perforation would have been missed. In this study, patients were not sub-grouped according to the presence of immunocompromising. Van de Wall et al. [25] found that the optimal CRP threshold distinguishing complicated from uncomplicated diverticulitis was $175 \mathrm{mg} / \mathrm{l}$, but $39 \%$ of patients with a complicated episode had a CRP below this threshold. Thus, a low CRP does not exclude complicated diverticulitis. Focusing on elderly

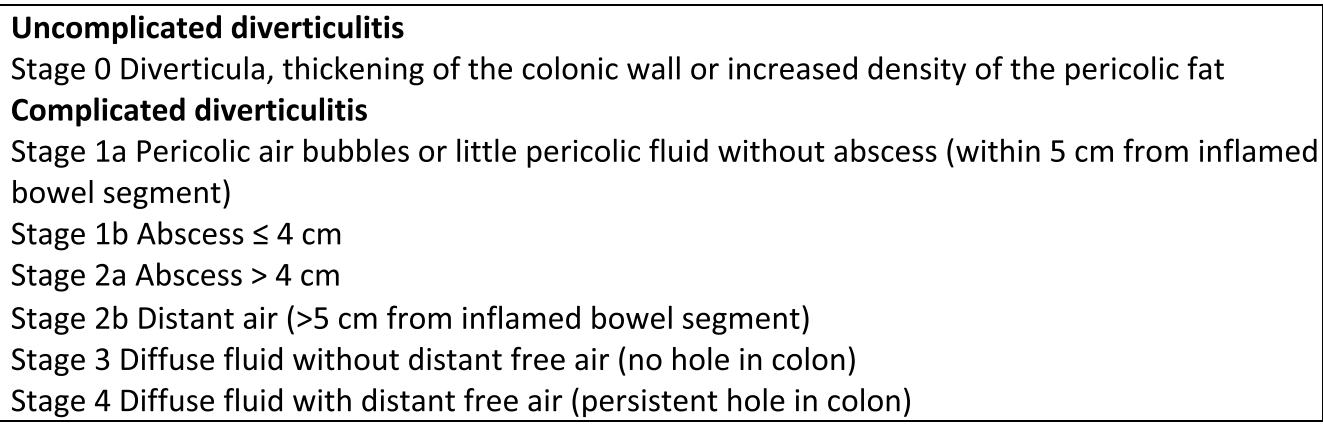

Fig. 1 WSES left colonic diverticulitis classification [17] 
patients, as previously reported, the clinical presentation of ALCD is more nuanced. Only $50 \%$ of patients older than 65 years with ALCD have pain in the lower quadrants of the abdomen, with only $17 \%$ having fever and $43 \%$ do not have a leucocytosis [4].

2. What is the optimum pathway for imaging in elderly patients with suspected acute left colonic diverticulitis? CT or US or both?

Statement 2.1 We suggest the use of CT-scan with IV-contrast in all elderly patients with suspected diverticulitis to confirm the diagnosis and to distinguish complicated from non-complicated diverticulitis. [Conditional recommendation, very lowquality evidence]

Statement 2.2 In elderly patients with suspected diverticulitis who cannot undergo CT-scan with IV-contrast (i.e. severe acute or chronic kidney disease or contrast allergy), we suggest the use of US, MRI or CT-scan without IV-contrast as alternative diagnostic approaches, according to resources availability. [Conditional recommendation, very low-quality evidence]

According to the WSES Guidelines $[13,14]$ in the general population, regarding $\mathrm{CT}$ scans of the abdomen and pelvis, this is indicated for all patients with suspected ALCD, but ultrasound (US) may be a useful alternative in the initial evaluation of patients. A stepup approach with $\mathrm{CT}$ performed after an inconclusive or negative US may be a safe approach for suspected acute diverticulitis in the general population $[13,14]$. US could be used to confirm or exclude other conditions which do not require CT. The reported sensitivity and specificity are 0.95 and $0.96-0.99$ for CT scan and 0.90 and $0.90-1.00$ for US, respectively $[18,19]$. In a meta-analysis by Andeweg et al [19], the pooled specificity of CT (96\% [95\% CI 90-100\%]) was significantly higher compared with US (90\% [95\% CI 86-94\%] $(p=0.04$; OR 2.46; 95\% CI 1.01-5.96) and an accurate diagnosis was made in $68 \%$ of patients with a CT scan and in $48 \%$ with US ( $p=0.002$, OR 2.6 ; $95 \% \mathrm{CI}$ 1.41-4.93). In the study by Nielsen et al [26] in cases of uncomplicated diverticulitis CT scan and US results were not comparable in $17 \%$ of cases (no diverticulitis on US in $14 \%$ and inconclusive US in $3 \%$ of cases) and in cases of complicated diverticulitis they were not comparable in $79 \%$ of cases (uncomplicated diverticulitis on US in $34 \%$, no diverticulitis on US in $28 \%$ and inconclusive US in 17\% of cases). The importance of CT imaging also emerged from the propensity score analysis by Shin et al in which the presence of distant intraperitoneal air on $\mathrm{CT}$ was the only significant parameter that correlated with operative management of colonic diverticulitis [27]. Focusing on elderly patients, among the 464 patients older than 80 years presenting to the ED with acute abdominal pain [28], 55\% had positive CT scans and $9 \%$ had diverticulitis. The clinical diagnosis obtained was clinically unsuspected prior to $\mathrm{CT}$ in $43 \%(p<0.05)$ with significant difficulty in diagnosing of diverticulitis $(p<0.01)$. Furthermore, CT results influenced treatment plans in $65 \%$ overall, surgical in $48 \%$ of these and medical in $52 \%$.

Hence, the importance of performing abdominal and pelvic CT scan with IV-contrast in all elderly patients with suspected ALCD (regardless of the value of leukocytes and of CRP) to exclude other diagnoses and to distinguish complicated from non-complicated diverticulitis, and to promptly plan the correct treatment.

The prevalence of chronic kidney disease in the US is $39.4 \%$ of persons aged $60+$ years versus 12.6 and $8.5 \%$ of persons aged $40-59$ years and $20-39$ years, respectively [29]. However, the high prevalence of kidney disease among elderly patients should not discourage CT scan execution with IV-contrast, because, usually, a prompt diagnosis and treatment in this frail population may justify the risk of contrast-induced acute kidney injury (CI-AKI).

Even if there is general agreement that chronic kidney disease represents the most significant independent predictor of CI-AKI [30], especially in the subset of patients with $\mathrm{eGFR} \leq 45 \mathrm{ml} / \mathrm{min}[31$ ], a recent meta-analysis of retrospective cohort studies of IV radiographic contrast failed to show a higher risk of CI-AKI after CT-scan in patients with chronic kidney disease [32].

This finding is consistent with other recent studies [33-37]. Clinicians should reassess the weight attributed to potential CI-AKI in their decision-making process. Furthermore, in addition to the patient-related risk factors for CI-AKI (advanced age, chronic kidney disease, diabetes mellitus, reduced effective circulating volume, congestive heart failure, anaemia, kidney transplant and concomitant nephrotoxic drugs), modifiable factors related to the procedure may play a role in the occurrence of CI-AKI. These include contrast media volume, route of contrast administration (intra-arterial versus intravenous), type of contrast media, and repeated procedures in a narrow temporal window [30]. Furthermore, the concomitant and crucial resuscitation with crystalloids and antibiotics would minimize the incidence of CI-AKI. 
Alternative diagnostic approaches, even if less accurate, in elderly patients with suspected ALCD who cannot undergo CT-scan with IV-contrast exist. They are US, MRI or CT-scan without IV-contrast. The reported sensitivity and specificity of MRI for ALCD are 0.98 and $0.70-0.78$, respectively [19]. However, MRI is rarely a feasible imaging modality in an urgent setting. Concerning unenhanced CT, in a study of 208 patients older than 75 years presenting to ED with acute abdominal pain, radiologists changed the diagnosis suspected by the emergency physicians in $59.1 \%$ of patients with unenhanced CT images only and in $61.2 \%$ of patients with both unenhanced and contrast-enhanced CT. The addition of contrast medium did not significantly improve the frequency of change in diagnosis $(p=0.746)$ [38]. However, the evidence is insufficient to recommend it as the first-choice examination. Furthermore, in a recent prospective study on patients with suspected diverticulitis, although unenhanced low-dose CT showed good sensitivity (98.6\%) for the detection of diverticulitis with excellent intermodality agreement, it had significantly lower sensitivity (61\%) for the detection of complications [39].

Recently, the use of point-of-care ultrasound (POCUS) by non-radiologists has dramatically increased. It could have a role in the diagnosis of acute diverticulitis and in those elderly patients who cannot undergo CT-scan $[40,41]$. Generally, abnormal ultrasound findings in ALCD include a thickened wall of more than $4 \mathrm{~mm}$, noncompressibility and loss of peristalsis. The layers of the colonic wall are usually preserved in diverticulitis compared with malignant tumours. Furthermore, POCUS may detect complications of diverticulitis which may include abscess, free intraperitoneal fluid, and free intraperitoneal air [40].

\section{Management}

3. What is the best treatment for uncomplicated diverticulitis (WSES stage 0) in elderly patients?

4. What is the best treatment for localized complicated diverticulitis without abscess (WSES stage 1a) diverticulitis in elderly patients?

Statement 3.1 We suggest that antibiotic therapy should be avoided in immunocompetent elderly patients with uncomplicated left colonic diverticulitis (WSES stage 0) without sepsis-related organ failures [Conditional recommendation, very lowquality of evidence]
Statement 4.1 We suggest antibiotic therapy administration for elderly patients with localized complicated left colonic diverticulitis with pericolic air bubbles or little pericolic fluid without abscess (WSES stage 1a). [Conditional recommendation, moderate quality of evidence]

According to the WSES guidelines [13, 14], antibiotic therapy can be avoided in immunocompetent patients with uncomplicated diverticulitis without systemic manifestations of infection. The AVOD trial [42] involved ten surgical departments in Sweden and one in Iceland and recruited 623 patients with a mean age of 57 years with CT-verified uncomplicated ALCD. Patients were randomized to treatment with (314 patients) or without (309 patients) antibiotics. Antibiotic treatment in patients with uncomplicated ALCD (without CT signs of abscess, fistula, or free air) neither accelerates recovery nor prevents complications or recurrences. The DIABOLO trial [43] included 528 patients with CT-proven, primary, left-sided, uncomplicated, ALCD (Hinchey 1a-1b) with age ranging from 48 to 64 years. Amoxicillin plus clavulanic acid $1.2 \mathrm{~g}$ four times daily intravenously for at least 48 hours (after which the route was switched to oral administration of 625 $\mathrm{mg}$ three times daily), for a first episode of CT-proven uncomplicated ALCD, demonstrated that observational treatment without antibiotics did not prolong recovery and could be considered appropriate in patients with uncomplicated diverticulitis. Even if no significant differences between Hinchey stages $1 \mathrm{a}$ and $1 \mathrm{~b}$ diverticulitis were found, it should be noted that the vast majority of patients included had a diagnosis of Hinchey stage 1a ALCD (90.1\% in the observational and $94 \%$ in the antibiotic-treated group) with only a small percentage of patients with Hinchey stage $1 \mathrm{~b}$ diverticulitis.

Omitting antibiotics in the treatment of uncomplicated ALCD did not result in more complicated diverticulitis, recurrent diverticulitis or sigmoid resections at longterm follow-up. As the DIABOLO trial was not powered for these secondary outcome measures, some uncertainty remains whether (small) non-significant differences could be true associations.

A systematic review and meta-analysis including 2469 patients (including elderly patients) with acute uncomplicated diverticulitis [44] showed that treatment of acute uncomplicated diverticulitis without antibiotics might be feasible with outcomes that are comparable to antibiotic treatment and with potential benefits for patients and the overall health economic system. 
Furthermore, antibiotic use in patients with acute uncomplicated diverticulitis seems to increase the length of hospital stay [45].

Finally, current evidence [46] does not support the administration of antibiotics to improve outcomes in carefully selected healthy patients with uncomplicated ALCD. However, this evidence comes from young patients (mean age in AVOD trial: 57; age range in DIABOLO trial: 48-64). For this reason, for elderly patients, the recommendation has very low-quality evidence. Further studies should help identify specific subpopulations of patients who would derive benefit from antibiotic therapy and help define appropriate antibiotic regimens and treatment durations that minimize cost, adverse effects, and risk of anti-microbial resistance. Due to the low event rate, it remains uncertain whether antibiotic treatment is necessary in cases with isolated pericolic gas, whether in young or elderly patients [14].

5. What Is the best treatment for left colonic diverticulitis with abscess (WSES 1b-2a) in elderly patients?

Statement 5.1 In elderly stable patients with an abscess from acute left colonic diverticulitis (WSES stage $1 \mathrm{~b}-2 \mathrm{a}$ ) and without peritonitis, we suggest the administration of a broad-spectrum antibiotic therapy. [Conditional recommendation, very low quality of evidences]

Statement 5.2 We suggest adding percutaneous drainage to antibiotic therapy in elderly patients with acute left colonic diverticulitis and an abscess larger than $4 \mathrm{~cm}$ (WSES stage 2a), when skills and facilities are available. Cultures from percutaneous drainage should be carried out to guide the antibiotic therapy. [Conditional recommendation, very low quality of evidences]

ALCD is associated with an abscess in nearly $20 \%$ of cases [47]. Current evidence is of poor quality with no randomized studies, and there are no studies focused on the elderly.

The rationale for percutaneous drainage is to remove the source of sepsis where antibiotics could fail to reach adequate concentrations, with a consequent failure of the non-operative treatment. As with all the non-operative management strategies, it is indicated only in patients who remain stable without septic shock, where prompt and adequate source control is mandatory.
The treatment with percutaneous drainage has been considered the preferred treatment option since its dissemination and generally, a size of $4 \mathrm{~cm}$ has been considered as the cut-off for the indication to drain [48-51].

In a systematic review of observational and retrospective studies, the treatment of abscess with a median size of $4 \mathrm{~cm}$ (range: $1.5-5-0 \mathrm{~cm}$ ) with antibiotic therapy alone failed in $18.7 \%$ of cases with a low mortality rate; percutaneous drainage of abscesses with a median size of $6.1 \mathrm{~cm}$ (range $4.6-8.7 \mathrm{~cm}$ ) failed in $21.1 \%$ [52]. Surgery is associated with a higher mortality rate in the elderly, and it is not the preferred first-line option in stable patients. Rather, it is reserved for failure of non-operative management.

A retrospective observational study from Finland addressed the treatment of diverticular abscesses larger than $40 \mathrm{~mm}$ with antibiotics only or with the addition of percutaneous drainage. The study results demonstrated that the outcomes were comparable with similar failure rates (44\% for antibiotics and 33\% for drainage) and similar morbidity and mortality; however, the study was retrospective and was not focused on the elderly [53]. In elderly patient, we suggest adding percutaneous drainage to antibiotic therapy in cases with an abscess larger than $4 \mathrm{~cm}$. When skills and facilities are not available, we recommend to consider transferring the patient to a higher level hospital.

6. What is the best treatment for elderly patients with acute diverticulitis with distant free intraperitoneal air and without diffuse fluid (WSES stage 2b)?

Statement 6.1: In elderly patients with acute left colonic diverticulitis and CT findings of distant intraperitoneal free air and no free fluid (WSES stage $2 \mathrm{~b}$ ) we suggest against non-operative management as a viable option. [Conditional recommendation, very low quality of evidences]

The presence of free abdominal air is generally considered as a surgical indication. The non-operative management in patients with abdominal free air without diffuse fluid and generalized peritonitis has been described in several studies [54-56]. WSES guidelines suggest a non-operative treatment only in selected patients with distant air and without diffuse fluid [14]. Non-operative management with antibiotic therapy was associated with a high failure rate, ranging from 10 to $43 \%$. It should be noted that the studies also included patients with pericolic free air and only a small 
proportion of patients with distant free air. None of the studies focused on the elderly and were retrospective, generating a very low-level quality of evidence. The available evidence does not support the indication for non-operative management in cases with distant abdominal free air in elderly patients, and surgical exploration is suggested.

7. What is the best treatment for elderly patients with acute diverticulitis and diffuse peritonitis (WSES stage 3-4)?

Statement 7.1 In elderly patients with acute left colonic diverticulitis and diffuse peritonitis (WSES stage 3-4) we recommend against non-operative management as a viable option. [Strong recommendation, very low quality of evidences]

Statement 7.2 In elderly patients with acute left colonic diverticulitis and diffuse peritonitis (WSES stage 3-4) we recommend prompt and effective source control surgery. [Strong recommendation, very low quality of evidences]

Patients with diffuse peritonitis are often critically unwell and require immediate fluid resuscitation, antibiotic treatment and surgery. Although perforated diverticulitis complicated by diffuse peritonitis has a low absolute prevalence, it has a high postoperative mortality, regardless of surgical technique [13].

8. When is a planned elective sigmoid resection indicated in elderly patients with left colonic diverticular disease?

Statement 8.1 We suggest against elective sigmoid resection after a conservatively treated episode of acute left colonic diverticulitis in asymptomatic elderly patients without stenosis, fistulae or recurrent diverticular bleeding. [Conditional recommendation, very low-quality evidence]

Statement 8.2 We suggest considering elective sigmoid resection after a conservatively treated episode of acute left colonic diverticulitis in high-risk elderly patients, such as immunocompromised patients (if fit for surgery). [Conditional recommendation, very low-quality evidence]
Statement 8.3 We suggest elective sigmoid resection in elderly patients (if fit for surgery) with left colonic diverticular disease complicated with stenosis, fistulae or recurrent diverticular bleeding. [Conditional recommendation, very low-quality evidence]

Statement 8.4 We suggest elective sigmoid resection in elderly patients (if fit for surgery) with very symptomatic left colonic diverticular disease, which compromise the quality of life. [Conditional recommendation, very low-quality evidence]

As reported above, according to most authors [7, 9, 12], the risk of recurrence after a first episode of ALCD in the elderly population is significantly lower than in younger patients. In observational studies [57] of younger patients, the reported risk of recurrent ALCD after a medically treated diverticular abscess was up to $60 \%$. In other studies on patients with a mean age of 60 years, the reported recurrence rate was $9-30 \%$ [58, 59], with only $2.7 \%$ requiring sigmoid resection with a stoma and no mortality reported [59].

Furthermore, in an article by Lidor et al., the postoperative mortality and morbidity was higher in the elderly population after urgent sigmoid resection for ALCD. However, the effect of age on postoperative mortality was more pronounced after elective surgery, where mortality rates ranged from $0.56 \%$ in patients $65-69$ years old up to $6.5 \%$ in patients older than 85 years old [60]. However, in the article, the causes of mortality were not reported. Furthermore, because many immunocompromised patients are operated on electively after conservative treatment while many immunocompetent patients are not, there can be a bias because more immunocompromised patients may have been operated on in an elective fashion.

As stated in the WSES Guidelines [13, 14], patientrelated factors and not the number of previous diverticulitis episodes should be considered in planning elective sigmoid resection in patients with ALCD treated non-operatively.

Thus, taking into account the low risk of recurrence after a first episode of diverticulitis in the elderly population, even in the presence of a medically treated diverticular abscess, with the high postoperative mortality and morbidity after elective surgery for diverticulitis in this population, a case-by-case balance of risks and benefits should be made. If, after a medically resolved episode of ALCD, the diverticular disease is asymptomatic or mildly symptomatic, we suggest against planning 
elective sigmoid resection. On the other hand, if there is very symptomatic disease or complicated by stenosis, fistulae, or recurrent diverticular bleeding, compromises the patient's quality of life, an elective sigmoid resection in patients who are fit for surgery could be considered.

Immunocompromised patients need to be evaluated carefully, which includes patients such as those with organ transplant and patients using corticosteroids. Because they are at increased risk of having complicated diverticulitis requiring emergency surgery [61-64], as stated in the WSES Guidelines [13, 14], after a conservatively treated episode of ALCD an elective sigmoid resection should be planned.

\section{Is endoscopic screening recommended for elderly} patients treated with non-operative management for acute left colonic diverticulitis?

Statement 9.1 We suggest planning early colonic evaluation in elderly patients after an episode of acute left colonic diverticulitis. [Conditional recommendation, very low-quality evidence]

A recent large retrospective study [65] on 932,860 patients with the first episode of diverticulitis showed that individuals are twice as likely to be diagnosed with colorectal cancer within one year of their first episode of acute diverticulitis compared with individuals without diverticulitis. The WSES guidelines $[13,14]$ recommended that endoscopic evaluation be performed after an episode of complicated ALCD to rule out colorectal cancer. However, the indication for colonoscopy is debated in patients with uncomplicated episodes. In a systematic review and meta-analysis performed by Meyer et al., they found a pooled prevalence of colorectal cancer of $1.3 \%$ (95\% CI $0.1-2.0 \%$ ) among 3834 patients with uncomplicated episodes [66, 67]. Similarly, a recent publication reported colorectal cancer prevalence to be $1.8 \%$ in a cohort of 227 patients with uncomplicated ALCD [68]. Furthermore, age older than 50 years was found to be a significant risk factor for advanced colonic neoplasia at follow-up colonoscopy after medically treated acute uncomplicated ALCD [69].

With this in mind, we suggest that in elderly patients, an early colonic evaluation (colonoscopy or CT colonography) after a conservatively treated episode of ALCD should be considered.

\section{Surgical technique}

10. Should laparoscopic peritoneal lavage and drainage be considered in elderly patients with acute diverticulitis?

Statement 10.1 In elderly patients with acute left colonic diverticulitis and acute peritonitis we suggest against laparoscopic lavage as the preferred surgical approach due to the higher risk of failure to control the source of sepsis. [Conditional recommendation, moderate quality evidences]

In case of diffuse purulent peritonitis (Hinchey III) laparoscopic peritoneal lavage has been proposed as alternative to colonic resection. Three randomized trials have been published addressing this topic, and several meta-analyses summarized their findings, focusing the interest for this potentially less invasive approach among the scientific community [70-78]. This minimally invasive approach with laparoscopic lavage has been demonstrated to have similar short-term mortality and similar long-term results [79]. However, patients treated with laparoscopic lavage had a considerably higher incidence of intra-abdominal abscesses and higher reoperation rates during the index admission due to inadequate source control. The mentioned studies did not include elderly patients, and therefore, the results cannot be easily generalized. Source control, in elderly and frail patients, must be the most important target in the treatment of ALCD, avoiding the risk of a second "septic hit" derived from incomplete treatment. Therefore, we suggest against laparoscopic lavage as the preferred technique in managing elderly patients with Hinchey III ALCD. This option could be considered in very selected, well-informed patients after considering the risks and the potential benefit of a lesser invasive procedure.

11. What is the best surgical procedure for elderly patients with perforated diverticulitis with generalized peritonitis: Hartmann resection or resection with primary anastomosis or damage control surgery?

Statement 11.1 We suggest that in elderly patients with perforated diverticulitis with generalized peritonitis, Hartmann operation and resection with primary anastomosis are both reasonable options. [Conditional recommendation, low-quality of evidence] 
Statement 11.2 We suggest that in elderly patients with perforated diverticulitis with generalized peritonitis and physiological derangement, Damage Control Surgery (emergency laparotomy, source control and application of open abdomen and abdominal vacuum-assisted closure) may be a viable option. [Conditional recommendation, very low quality of evidence]

According to the WSES guidelines [13, 14], the Hartmann technique is still recommended for patients with diffuse peritonitis who are critically unwell or have numerous comorbidities. Primary resection with anastomosis with or without a diverting stoma may be performed in clinically stable patients with no comorbidities. For clinically unstable patients with diverticular peritonitis (severe sepsis/septic shock), a damage control surgery technique may be recommended.

Despite limitations due to the lack of strong evidence, some authors $[80,81]$ have attempted to propose a treatment strategy for various clinical scenarios of ALCD in the general population.

A meta-analysis by Halim et al. [82] including 3,546 patients with Hinchey III and IV diverticulitis comparing Hartmann procedure (HP) and resection and primary anastomosis (R-PA) with or without faecal diversion, included 22 observational studies and 3 RCTs. The overall mortality in the HP group was $10.8 \%$ across the observational studies and $9.4 \%$ in the RCTs. The mortality rates in the R-PA group, $8.2 \%$ in the observational studies and $4.3 \%$ in the RCTs, were lower than those in the HP group. They found a $40 \%$ lower mortality rate in the R-PA group than in the HP (OR 0.60, 95\% CI 0.38-0.95, $p=0.03$ ) when analysing the observational studies. However, meta-analysis of the three RCTs did not demonstrate any difference in mortality.

Another recent meta-analysis [83] found that R-PA was associated with better short- and long-term outcomes at the cost of significantly longer operating time at emergency surgery. No subgroup analyses based on age were made, nor specific studies on elderly patients.

A recent large retrospective study [84] on 10780 urgent/emergent colectomies for patients with diverticulitis showed that postoperative mortality was twofold greater when non-colorectal surgeons performed R-PA vs HP (15\% vs $7.4 \% ; p<0.001)$ and 1.4 times greater among non-colorectal surgeons than among colorectal surgeons $(7.5 \%$ vs $5.3 \% ; p=0.04)$. On multivariable logistic regression (adjusting for patient demographics/characteristics, year, hospital academic status and surgeon training) R-PA remained associated with increased mortality (OR 2.7; 95\% CI 1.7-4.4; $p<0.001$ ), complications (OR 1.8; 95\% CI 1.3-2.5; $p<0.001$ ), and reoperation (OR 3.4; 95\% CI 1.8-6.3; $p<0.001$ ). However, the patients included within this study were not randomly assigned to each treatment arm. Then, patients who were selected to receive a R-PA may be assumed to have been less ill on presentation.

The recent WSES guidelines covering the management of intra-abdominal sepsis [85] stated that there is insufficient evidence to advocate for damage control surgery (DCS) as a general strategy in patients with secondary peritonitis. On the other hand, even though it comes with a low grade of recommendation, the same guidelines suggest that DCS may be an option in selected significantly physiologically deranged patients with ongoing sepsis. There are few case series about DCS emergency laparotomy with the temporary application of abdominal vacuumassisted closure (VAC) for perforated diverticulitis, including also elderly patients [86-90]. The Perathoner et al. [87] series included 15 patients (median age 68 years, range 35-89) who underwent DCS with lavage, limited resection of the diseased colonic segment, abdominal VAC, and second-look operation. Of these, nine had intestinal continuity restored during a second-look operation, with one patient developing anastomotic leakage. Sohn et al. [86] reported 58 patients (median age 70 years, range $30-92$ ) who underwent DCS for Hinchey III (81\%) and Hinchey IV (19\%) ALCD, reporting a secondary bowel restoration rate of $83 \%$ with a $24 \%$ of loop ileostomy rate. Overall, $10 \%$ of patients experienced an anastomotic leak that eventually required the creation of a stoma: at the end of the hospital stay, they documented a $50 \%$ rate of patients without a stoma. DCS led to a significantly reduced stoma rate after the initial hospital stay without an increased risk of postoperative morbidity [91].The study by Kafka-Ritsh et al. [88] included 51 patients (median age 69 years, range $28-87$ ) with diverticular peritonitis treated with DCS with a rate of bowel restoration at the second-look operation of $76 \%$ and with a $11 \%$ rate of loop ileostomy. Their fistula rate was $13 \%$. Tartaglia et al. reported 34 patients (13 Hinchey III and 21 Hinchey IV) with a mean age of 66.9 years and a Mannheim Peritonitis Index (MPI) of $25.12(\mathrm{SD} \pm 6.28)$ treated with DCS: 24 patients $(71 \%)$ had restoration of bowel continuity, 10 (29\%) patients had an end colostomy (HP) and 1 patient had an anastomotic leak. The mortality rate was $12 \%$. 
A recent retrospective study [92], including 290 patients who underwent urgent laparotomy for nontrauma peritonitis, compared DCS and definitive initial surgical management. They found that DCS in severe non-trauma peritonitis patients was feasible and safe as surgical strategy management without increasing mortality, length hospital of stay, stoma rate or complications.

There are insufficient data focused on elderly patients to recommend or suggest R-PA instead of HP. Both are reasonable options in cases of perforated diverticulitis with generalized peritonitis. The choice could be based on the patient's current clinical status and pre-existing conditions (degree of autonomy, comorbidity, medication taken). DCS could be a viable option in cases with severe physiological derangement.

12. Should emergency laparoscopic sigmoidectomy (ELS) be considered in elderly patients with perforated diverticulitis with diffuse peritonitis?

Statement 12.1 We suggest that in stable elderly patients with perforated diverticulitis with diffuse peritonitis emergency laparoscopic sigmoidectomy can be performed by experienced laparoscopic surgeons [Conditional recommendation, very low quality of evidence]

Laparoscopic sigmoidectomy for diverticulitis was initially confined to the elective setting. However, in stable patients and if performed by experienced hands, ELS may also be feasible in Hinchey III and IV ALCD with reported good outcomes [93-95]. In a propensity score-matched cohort of patients with perforated diverticulitis [96], ELS was superior to open sigmoidectomy with regard to postoperative morbidity and hospital stay. However, these results should be interpreted with caution as the cohort consists of selected patients with more favourable characteristics and young age (mean age 56 years), and surgery was performed by experienced gastrointestinal surgeons.

\section{Antibiotic therapy}

13. What is the best anti-microbial regimen for elderly patients with localized complicated diverticulitis?

14. What is the best anti-microbial regimen for elderly patients with perforated diverticulitis with diffuse peritonitis?
Statement 13.1 In elderly patients with localized complicated diverticulitis the empirically designed anti-microbial regimen depends on the underlying clinical condition of the patient, the pathogens presumed to be involved, and the risk factors indicative of major resistance patterns. [Conditional recommendation, very low quality of evidence]

Statement 14.1 In elderly patients with perforated diverticulitis with diffuse peritonitis the empirically designed anti-microbial regimen depends on the underlying clinical condition of the patient, the pathogens presumed to be involved, and the risk factors indicative of major resistance patterns. [Conditional recommendation, very low quality per indirectness]

According to the WSES guidelines [12, 14], the empirically planned antimicrobial regimen is based on the patient's underlying clinical condition, the microorganisms suspected of being involved, and risk factors for significant resistance patterns. The principles of empiric antibiotic treatment should be developed based on the most commonly isolated microorganisms, while also taking into account the local trend of antibiotic resistance [85]. The presence of anaerobes and Gramnegative bacteria in the lower gastrointestinal tract should be taken into account while choosing empirical therapy for acute diverticulitis. In addition, quinolone and carbapenem resistance, as well as the presence of ESBL-producing bacteria in the local environment and the location of recent travel, should always be taken into account.

Because inoculation in a healthcare facility, corticosteroid usage, organ transplantation, baseline pulmonary or hepatic disease, and past anti-microbial therapy are the most relevant factors in predicting the presence of resistant bacteria, elderly individuals frequently fall into this category. One of the factors that is significantly linked to poor outcomes in critically ill patients is an ineffective or inadequate antimicrobial treatment. In patients with organ dysfunction and septic shock, broad empiric antimicrobial therapy should be started as soon as possible [97].

Furthermore, for individuals at risk for resistant bacteria, intraperitoneal samples for microbiological evaluation are always indicated from the site of infection. When the findings of microbiological tests are available, the patient should be reviewed, and antimicrobial de-escalation or withdrawal should be considered [98]. 
15. When should discontinuation of anti-microbial treatment be considered?

Statement 15.1 In elderly patients with complicated diverticulitis a short course of antibiotic therapy (3-5 days) after adequate source control is a reasonable option. [Conditional recommendation, moderate quality of evidence]

Statement 15.2 In elderly patients with complicated diverticulitis who have ongoing signs of peritonitis or systemic illness (ongoing infection) beyond 5 to 7 days of antibiotic treatment, further diagnostic investigation is indicated. [Conditional recommendation, low quality of evidence]

The outcomes after 4 days of antibiotic therapy were similar to those following a longer course of antibiotics in patients with intraabdominal infections who completed an acceptable source control regimen [99, 100].

Although discontinuation of anti-microbial treatment should be based on clinical criteria, a 4-6 day period of postoperative anti-microbial therapy in complicated ALCD is suggested if source control has been adequate [12]. Patients who have persistent symptoms of peritonitis or systemic sickness (ongoing infection) after 5 to 7 days of antibiotic treatment should be re-evaluated.

\section{Conclusion}

After the publication of the WSES guidelines for the management of acute left-sided colonic diverticulitis in the emergency setting $[13,14]$ for the diagnosis and management of ALCD in the general population, the present guidelines represent, to the best of our knowledge, the first clinical guidelines for diagnosis and management of ALCD in elderly patients ( $>65$ years of age).

During the $1^{\circ}$ Pisa Workshop of Acute Care \& Trauma Surgery held in Pisa (Italy) in September 2019 with the collaboration of WSES, SICG, ACOI, SICUT, AcEMC and SIFIPAC, a panel of experts participated in a Consensus Conference where three panel-members presented a number of statements, which were developed for each of the four topics regarding the diagnosis and management of ALCD in elderly patients, formulated according to the GRADE system: Diagnosis, Management, Surgical Technique, Antibiotic Therapy. The statements were then voted (Fig. 2), eventually modified and finally approved by the participants to The Consensus Conference (Table 1).

After reaching consensus on each of the above-mentioned statements, the Scientific Committee members developed the following algorithm for the diagnosis and management of ALCD in Elderly Patient, reported in Fig. 3.

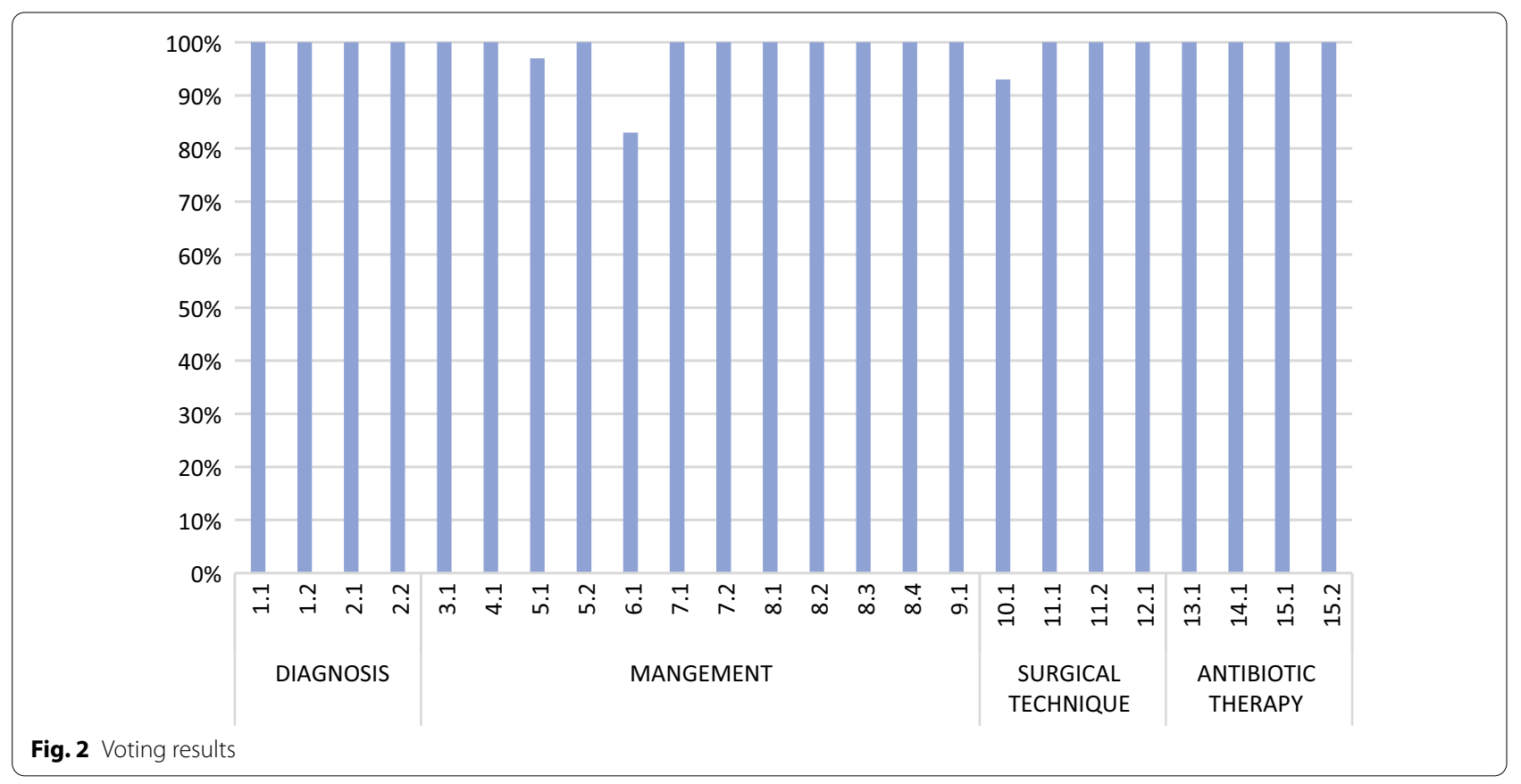


Table 1 Summary of recommendations

Diagnosis

Statement 1.1 In the elderly population, we suggest against basing the diagnosis of acute left colonic diverticulitis on only patient clinical signs, symptoms and laboratory tests. [Conditional recommendation, very low-quality evidence]

Statement 1.2 We suggest that elderly patients presenting with abdominal guarding or pain in the lower left abdomen on physical examination undergo appropriate imaging for suspected diverticulitis, regardless of the value of leukocytes and of C-reactive protein (CRP). [Conditional recommendation, very low-quality evidence]

Statement 2.1 We suggest the use of CT-scan with IV-contrast in all elderly patients with suspected diverticulitis to confirm the diagnosis and to distinguish complicated from non-complicated diverticulitis. [Conditional recommendation, very low-quality evidence]

Statement 2.2 In elderly patients with suspected diverticulitis who cannot undergo CT-scan with IV-contrast (i.e. severe acute or chronic kidney disease or contrast allergy), we suggest the use of US, MRI or CT-scan without IV-contrast as alternative diagnostic approaches, according to resources availability. [Conditional recommendation, very low-quality evidence]

Management

Statement 3.1 We suggest that antibiotic therapy should be avoided in immunocompetent elderly patients with uncomplicated left colonic diverticulitis (WSES stage 0) without sepsis-related organ failures [Conditional recommendation, very low-quality of evidence]

Statement 4.1 We suggest antibiotic therapy administration for elderly patients with localized complicated left colonic diverticulitis with pericolic air bubbles or little pericolic fluid without abscess (WSES stage 1a). [Conditional recommendation, moderate quality of evidence]

Statement 5.1 In elderly stable patients with an abscess from acute left colonic diverticulitis (WSES stage 1b-2a) and without peritonitis, we suggest the administration of a broad-spectrum antibiotic therapy. [Conditional recommendation, very low quality of evidences]

Statement 5.2 We suggest adding percutaneous drainage to antibiotic therapy in elderly patients with acute left colonic diverticulitis and an abscess larger than $4 \mathrm{~cm}$ (WSES stage 2a), when skills and facilities are available. Cultures from percutaneous drainage should be carried out to guide the antibiotic therapy. [Conditional recommendation, very low quality of evidences]

Statement 6.1: In elderly patients with acute left colonic diverticulitis and CT findings of distant intraperitoneal free air and no free fluid (WSES stage 2b), we suggest against non-operative management as a viable option. [Conditional recommendation, very low quality of evidences]

Statement 7.1 In elderly patients with acute left colonic diverticulitis and diffuse peritonitis (WSES stage 3-4), we recommend against non-operative management as a viable option. [Strong recommendation, very low quality of evidences]

Statement 7.2 In elderly patients with acute left colonic diverticulitis and diffuse peritonitis (WSES stage 3-4), we recommend prompt and effective source control surgery. [Strong recommendation, very low quality of evidences]

Statement 8.1 We suggest against elective sigmoid resection after a conservatively treated episode of acute left colonic diverticulitis in asymptomatic elderly patients without stenosis, fistulae or recurrent diverticular bleeding. [Conditional recommendation, very low-quality evidence]

Statement 8.2 We suggest to consider elective sigmoid resection after a conservatively treated episode of acute left colonic diverticulitis in high-risk elderly patients, such as immunocompromised patients (if fit for surgery). [Conditional recommendation, very-low quality evidence]

Statement 8.3 We suggest elective sigmoid resection in elderly patients (if fit for surgery) with left colonic diverticular disease complicated with stenosis, fistulae or recurrent diverticular bleeding. [Conditional recommendation, very low-quality evidence]

Statement 8.4 We suggest elective sigmoid resection in elderly patients (if fit for surgery) with very symptomatic left colonic diverticular disease which compromise the quality of life. [Conditional recommendation, very low-quality evidence]

Statement 9.1 We suggest planning early colonic evaluation in elderly patients after an episode of acute left colonic diverticulitis. [Conditional recommendation, very low-quality evidence]

surgical technique

Statement 10.1 In elderly patients with acute left colonic diverticulitis and acute peritonitis, we suggest against laparoscopic lavage as the preferred surgical approach due to the higher risk of failure to control the source of sepsis. [Conditional recommendation, moderate quality evidences]

Statement 11.1 We suggest that in elderly patients with perforated diverticulitis with generalized peritonitis Hartmann operation and resection with primary anastomosis are both reasonable options. [Conditional recommendation, low-quality of evidence]

Statement 11.2 We suggest that in elderly patients with perforated diverticulitis with generalized peritonitis and physiological derangement, Damage Control Surgery (emergency laparotomy, source control and application of open abdomen and abdominal vacuum-assisted closure) may be a viable option. [Conditional recommendation, very low quality of evidence]

Statement 12.1 We suggest that in stable elderly patients with perforated diverticulitis with diffuse peritonitis emergency laparoscopic sigmoidectomy can be performed by experienced laparoscopic surgeons [Conditional recommendation, very low quality of evidence]

Antibiotic therapy

Statement 13.1 In elderly patients with localized complicated diverticulitis the empirically designed anti-microbial regimen depends on the underlying clinical condition of the patient, the pathogens presumed to be involved, and the risk factors indicative of major resistance patterns. [Conditional recommendation, very low quality of evidence]

Statement 14.1 In elderly patients with perforated diverticulitis with diffuse peritonitis the empirically designed anti-microbial regimen depends on the underlying clinical condition of the patient, the pathogens presumed to be involved, and the risk factors indicative of major resistance patterns. [Conditional recommendation, very low quality per indirectness]

Statement 15.1 In elderly patients with complicated diverticulitis a short course of antibiotic therapy (3-5 days) after adequate source control is a reasonable option. [Conditional recommendation, moderate quality of evidence]

Statement 15.2 In elderly patients with complicated diverticulitis who have ongoing signs of peritonitis or systemic illness (ongoing infection) beyond 5 to 7 days of antibiotic treatment, further diagnostic investigation is indicated. [Conditional recommendation, low quality of evidence] 


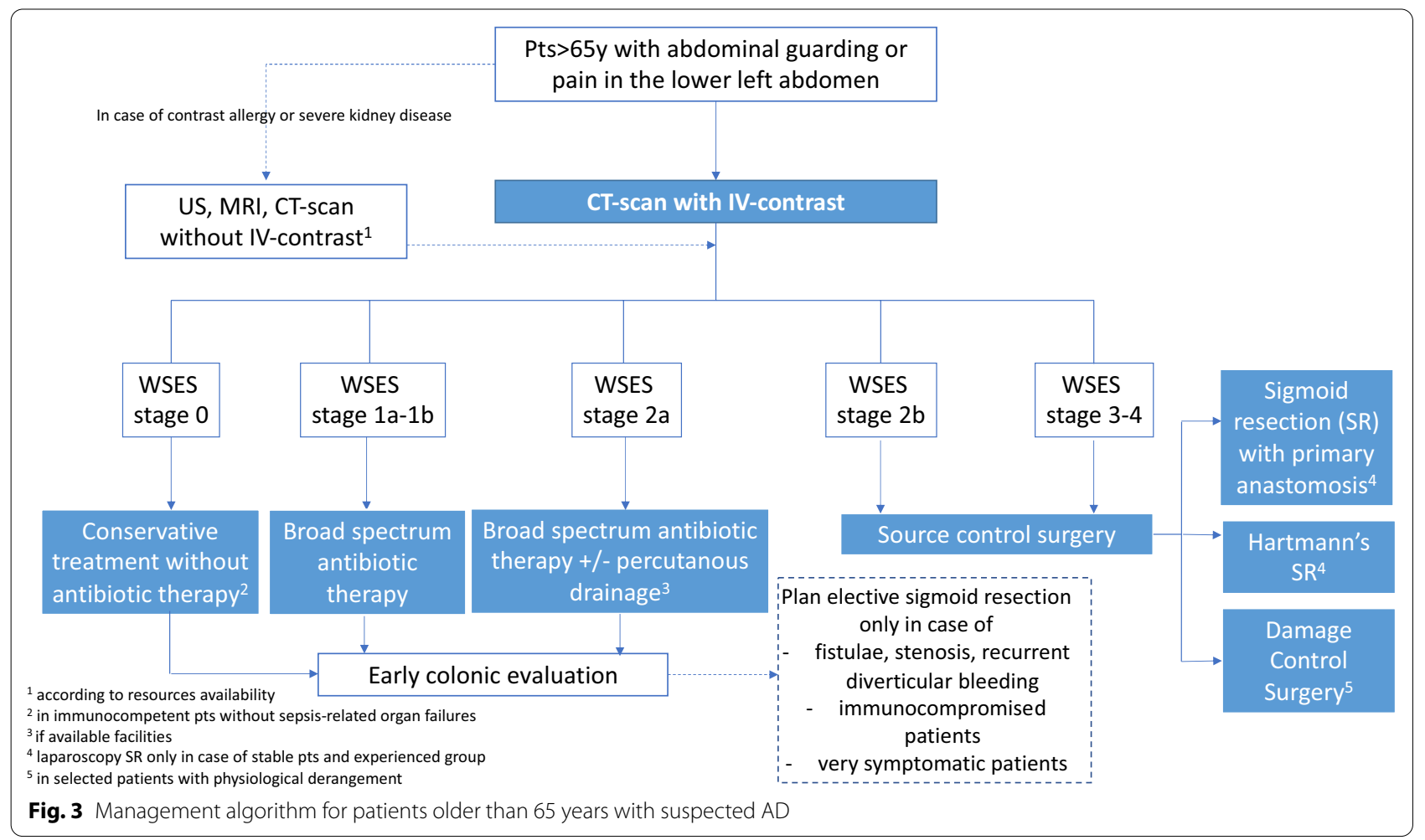

Unfortunately, due to the lack of high-quality studies focusing on elderly patients and to the heterogeneity of the existing studies in the definition of an age cut-off for the characterization of elderly patient, according to the GRADE methodology, all statements are based on lowor very low-quality evidence.

\section{Abbreviations}

ALCD: Acute left colonic diverticulitis; WSES: World Society of Emergency Surgery; SICG: Italian Society of Geriatric Surgery; ACOI: Italian Hospital Surgeons Association; SICUT: Italian Emergency Surgery and Trauma Association; AcEMC: Academy of Emergency Medicine and Care; SIFIPAC: Italian Society of Surgical Pathophysiology; SUDD: Symptomatic uncomplicated diverticular disease; NPV: Negative predictive value; ED: Emergency department; CT: Computed tomography; US: Ultrasound; CI-AKI: Contrast-induced acute kidney injury; MRI: Magnetic resonance imaging; IV: Intravenous; POCUS: Point-of-care ultrasound; HR: Hartmann procedure; R-PA: Resection and primary anastomosis; RCT: Randomized controlled trial; DCS: Damage control surgery; VAC: Vacuumassisted closure; ELS: Emergency laparoscopic sigmoidectomy.

\section{Acknowledgements}

Not applicable.

\section{Authors' contributions}

$\mathrm{PF}, \mathrm{MC}$ and $\mathrm{LA}$ have made substantial contributions to the conception and design of the work; FC, FG, AP, FM, BA, GS, MS, FM, AP, DT, PC, NA, YK, CP, MZ, AL, MT, LC, FDM; MI, EEM, ABP, MS, VA, GF, DGW, DD, FMAZ, IW, AWK, MP, NP, ET, RTB, RVM, RJD, JK, VGS, ACM, GA, SM, EP, BDS, MC, WB, GLB, FC and LA have drafted the work or substantively revised it; all authors approved the submitted version (and any substantially modified version that involves the author's contribution to the study); all authors agreed both to be personally accountable for the author's own contributions and to ensure that questions related to the accuracy or integrity of any part of the work, even ones in which the author was not personally involved, are appropriately investigated, resolved, and the resolution documented in the literature. All authors read and approved the final manuscript.

\section{Funding}

This research received no specific grant from any funding agency in the public, commercial or not-for-profit sectors.

\section{Availability of data and materials}

Not applicable.

\section{Declarations}

Ethics approval and consent to participate

Not applicable.

\section{Consent for publication}

Not applicable.

\section{Competing interests}

The authors declare that they have no competing interests.

\section{Author details}

${ }^{1}$ IRCCS Policlinico San Matteo Foundation, General Surgery, Pavia, Italy. ${ }^{2}$ General Surgery Department, School of Medicine and Surgery, Milano-Bicocca University, Monza, Italy. ${ }^{3}$ Emergency Surgery Unit, State University of Pisa, Cisanello Hospital, Pisa, Italy. ${ }^{4}$ Department of Surgery and Transplants, AOU San Giovanni di Dio and Ruggi d'Aragona, University of Salerno, Fisciano, Italy. ${ }^{5}$ Geriatrics Unit, Department of Clinical and Experimental Medicine, University of Pisa, Pisa, Italy. ${ }^{6}$ Department of Clinical Medicine and Surgery, University of Naples "Federico II", Naples, Italy. ${ }^{7}$ Emergency Surgery and Trauma, Fondazione Policlinico Universitario "A. Gemelli" IRCCS, Rome, Italy. ${ }^{8}$ Department of Surgery, Macerata Hospital, Macerata, Italy. ${ }^{9}$ Division of Infectious 
Diseases, Department of Clinical and Experimental Medicine, University of Pisa, Pisa, Italy. ${ }^{10}$ Department of Surgery, S. Anna University Hospital and University of Ferrara, Ferrara, Italy. ${ }^{11}$ Medical School, General Surgery and Surgical Specialties Unit, S. Maria University Hospital University of Perugia, Terni, Italy. ${ }^{12}$ Division of General Surgery, Rambam Health Care Campus, Haifa, Israel. ${ }^{13}$ Emergency Room Brescia Spedali Civili General Hospital, Brescia, Italy. ${ }^{14}$ Department of Robotic and Emergency Surgery, Manzoni Hospital, ASST Lecco, Lecco, Italy. ${ }^{15}$ Abdominal Center, Helsinki University Hospital and University of Helsinki, Helsinki, Finland. ${ }^{16}$ Department of Management, Lincoln International Business School, University of Lincoln, Lincoln, UK. ${ }^{17}$ Alma Mater Studiorum University, Bologna, Italy. ${ }^{18}$ Department of Surgery, University of Colorado, Denver Health Medical Center, Denver, CO, USA. ${ }^{19}$ Department of Surgery, University of Pittsburgh School of Medicine, UPMC-Presbyterian, Pittsburgh, PA, USA. ${ }^{20}$ Donegal Clinical Research Academy, Emergency Surgery Outcome Project, Letterkenny University Hospital, Donegal, Ireland. ${ }^{21}$ Intensive Care Unit, Bufalini Hospital, Cesena, Italy. ${ }^{22}$ Surgery Department, Faculdade de Ciências Médicas (FCM), Unicamp Campinas, Campinas, SP, Brazil. ${ }^{23}$ Department of General Surgery, Royal Perth Hospital, Perth, Australia. ${ }^{24}$ Department of Surgery, Royal Infirmary of Edinburgh, Edinburgh, UK. ${ }^{25}$ Department of Surgery, College of Medicine and Health Sciences, UAE University, Al-Ain, United Arab Emirates. ${ }^{26}$ Department of Minimal Access and General Surgery, Government Gousia Hospital, Sringar, Kashmir, India. ${ }^{27}$ General, Acute Care, Abdominal Wall Reconstruction, and Trauma Surgery, Foothills Medical Centre, Calgary, AB, Canada. ${ }^{28}$ 3Rd Department of Surgery, Attiko Hospital, MSC "Global Health-Disaster Medicine", National and Kapodistrian University of Athens (NKUA), Athens, Greece. ${ }^{29}$ General Surgery, Dr Sulaiman Al Habib/ Alfaisal University, Riyadh, Saudi Arabia. ${ }^{30}$ Department of Surgery, Radboud University Medical Centre, Nijmegen, The Netherlands. ${ }^{31}$ Department of Surgery, University of Washington, Seattle, WA, USA. ${ }^{32}$ Cambridge Colorectal Unit, Addenbrooke's Hospital, Cambridge University Hospitals NHS Foundation Trust, Cambridge, UK. ${ }^{33}$ Department of Surgery, Assia Medical Group, Tel Aviv University Sackler School of Medicine, Tel Aviv, Israel. ${ }^{34}$ Department of General Surgery, Tan Tock Seng Hospital, Singapore, Singapore. ${ }^{35}$ Faculty of Health Sciences, University of Buea, Buea, Cameroon. ${ }^{36}$ Department of Surgery, University Hospital Centre, Zagreb, Croatia. ${ }^{37}$ General Surgery I, ASST Papa Giovanni XXIII Hospital, Bergamo, Italy. ${ }^{38}$ Department of General and Metabolic Surgery, Poissy and Saint Germain en Laye Hospitals, Poissy, France. ${ }^{39}$ Trauma Surgery Department, Scripps Memorial Hospital, La Jolla, CA, USA. ${ }^{40}$ Department of General Surgery, ASST Cremona, University of Brescia, Cremona, Italy. ${ }^{41}$ General and Emergency Surgery Department, Bufalini Hospital, AUSL Romagna, Cesena, Italy.

Received: 28 July 2021 Accepted: 27 November 2021

Published online: 21 January 2022

\section{References}

1. Rugarli C, et al. Rugarli Medicina Interna Sistematica, 7th ed. Edra; 2015.

2. Ubaldi E, Grattagliano I, Lapi F, Pecchioli S, Cricelli C. Overview on the management of diverticular disease by Italian General Practitioners. Dig Liver Dis. 2019;51 (1):63-7. https://doi.org/10.1016/j.dld.2018.07.015.

3. Binda GA, et al. Trends in hospital admission for acute diverticulitis in Italy from 2008 to 2015. Tech Coloproctol. 2018;22(8):597-604. https:// doi.org/10.1007/s10151-018-1840-z.

4. Lizardi-Cervera J, Vázquez-Elizondo G, Becerra-Laparra I, Chávez-Tapia $\mathrm{N}$, Noriega-lriondo MF, Uribe M. Estudio comparativo de la presentación clínica y complicaciones de la diverticulitis aguda colónica en pacientes mayores y menores de 65 años. Rev Gastroenterol Mex. 2008;73(3):153-8.

5. Urabe $\mathrm{M}$, et al. Distinct clinical factors in hospitalized patients with diverticular bleeding and diverticulitis. Digestion. 2019;99(3):239-46. https://doi.org/10.1159/000491875.

6. Sirinthornpunya S. Characteristics and treatment outcomes of colonic diverticulitis in hospitalized patients in Thailand. J Med Assoc Thail. 2016;99:S136-46.

7. Katz LH, Guy DD, Lahat A, Gafter-Gvili A, Bar-Meir S. Diverticulitis in the young is not more aggressive than in the elderly, but it tends to recur more often: systematic review and meta-analysis. J Gastroenterol Hepatol. 2013;28(8):1274-81. https://doi.org/10.1111/jgh.12274.
8. Manabe $\mathrm{N}$, et al. Characteristics of colonic diverticulitis and factors associated with complications: a Japanese multicenter, retrospective, cross-sectional study. Dis Colon Rectum. 2015;58(12):1174-81. https:// doi.org/10.1097/DCR.0000000000000488.

9. Van De Wall BJM, Poerink JA, Draaisma WA, Reitsma JB, Consten ECJ, Broeders IAMJ. Diverticulitis in young versus elderly patients: a metaanalysis. Scand J Gastroenterol. 2013;48(6):643-51. https://doi.org/10. 3109/00365521.2012.758765.

10. Ukkonen M, Karlsson S, Laukkarinen J, Rantanen T, Paajanen H. Severe sepsis in elderly patients undergoing gastrointestinal surgery-a prospective multicenter follow-up study of finnish intensive care units. J Gastrointest Surg. 2016;20(5):1028-33. https://doi.org/10.1007/ s11605-016-3076-4.

11. Lidsky ME, Thacker JKM, Lagoo-Deenadayalan SA, Scarborough JE. Advanced age is an independent predictor for increased morbidity and mortality after emergent surgery for diverticulitis. Surgery (United States). 2012;152(3):465-72. https://doi.org/10.1016/j.surg.2012.06.038.

12. Lidor AO, Segal JB, Wu AW, Yu Q, Feinberg R, Schneider EB. Older patients with diverticulitis have low recurrence rates and rarely need surgery. Surgery. 2011;150(2):146-53. https://doi.org/10.1016/j.surg. 2011.05.006.

13 Sartelli M, et al. WSES Guidelines for the management of acute left sided colonic diverticulitis in the emergency setting. World J Emerg Surg. 2016. https://doi.org/10.1186/s13017-016-0095-0.

14. Sartelli M, et al. 2020 update of the WSES guidelines for the management of acute colonic diverticulitis in the emergency setting. World J Emerg Surg. 2020;15(1):1-18. https://doi.org/10.1186/ s13017-020-00313-4.

15 Pisano M, et al. 2017 WSES and SICG guidelines on acute calcolous cholecystitis in elderly population. World J Emerg Surg. 2019;7:1-16.

16 Guyatt GH. GRADE : an emerging consensus on rating quality of evidence and strength of recommendations. BMJ. 2008;336:924-6.

17. Sartelli M, et al. A proposal for a CT driven classification of left colon acute diverticulitis. World J Emerg Surg. 2015;10:1-11.

18. Toorenvliet BR, Bakker RFR, Breslau PJ, Merkus JWS, Hamming JF. Colonic diverticulitis: a prospective analysis of diagnostic accuracy and clinical decision-making. Colorectal Dis. 2010;12(3):179-86. https://doi. org/10.1111/j.1463-1318.2009.01778.x.

19. Andeweg CS, Wegdam JA, Groenewoud J, Van Der Wilt GJ, Van Goor H, Bleichrodt RP. Toward an evidence-based step-up approach in diagnosing diverticulitis. Scand J Gastroenterol. 2014;49(7):775-84. https://doi. org/10.3109/00365521.2014.908475.

20. Laurell H, Hansson LE, Gunnarsson U. Acute diverticulitis-clinical presentation and differential diagnostics. Colorectal Dis. 2007;9(6):496-501. https://doi.org/10.1111/j.1463-1318.2006.01162.x.

21. Laméris W, et al. A clinical decision rule to establish the diagnosis of acute diverticulitis at the emergency department. Dis Colon Rectum. 2010;53(6):896-904. https://doi.org/10.1007/DCR.0b013e3181d98d86.

22. Bolkenstein $\mathrm{HE}$, van de Wall BJ, Consten EC, van der Palen J, Broeders IA, Draaisma WA. Development and validation of a diagnostic prediction model distinguishing complicated from uncomplicated diverticulitis. Scand J Gastroenterol. 2018;53(10-11):1291-7. https://doi.org/10.1080/ 00365521.2018 .1517188$.

23. Longstreth GF, et al. Acute diverticulitis: Demographic, clinical and laboratory features associated with computed tomography findings in 741 patients. Aliment Pharmacol Ther. 2012;36(9):886-94. https://doi. org/10.1111/apt.12047.

24. Käser SA, Fankhauser G, Glauser PM, Toia D, Maurer CA. Diagnostic value of inflammation markers in predicting perforation in acute sigmoid diverticulitis. World J Surg. 2010;34(11):2717-22. https://doi.org/10. 1007/s00268-010-0726-7.

25. van de Wall BJM, Draaisma WA, van der Kaaij RT, Consten ECJ, Wiezer MJ, Broeders IAMJ. The value of inflammation markers and body temperature in acute diverticulitis. Colorectal Dis. 2013;15(5):621-6. https:// doi.org/10.1111/codi.12072.

26. Nielsen $\mathrm{K}$, et al. The limited role of ultrasound in the diagnostic process of colonic diverticulitis. World J Surg. 2014;38(7):1814-8. https://doi.org/ 10.1007/s00268-013-2423-9.

27. Shin S, Kim D, Kang UR, Yang CS. Impact of CT imaging on predicting the surgical management of acute diverticulitis. Ann Surg Treat Res. 2018;94(6):322-9. https://doi.org/10.4174/astr.2018.94.6.322. 
28. Gardner CS, Jaffe TA, Nelson RC. Impact of CT in elderly patients presenting to the emergency department with acute abdominal pain. Abdom Imaging. 2015;40(7):2877-82. https://doi.org/10.1007/ s00261-015-0419-7.

29. Coresh J, Astor BC, Greene T, Eknoyan G, Levey AS. Prevalence of chronic kidney disease and decreased kidney function in the adult US population: Third National Health and Nutrition Examination Survey. Am J Kidney Dis. 2003;41(1):1-12. https://doi.org/10.1053/ajkd.2003. 50007.

30. Pistolesi $\bigvee$, et al. Contrast medium induced acute kidney injury: a narrative review. J Nephrol. 2018;31(6):797-812. https://doi.org/10.1007/ s40620-018-0498-y.

31. McDonald JS, McDonald RJ, Williamson EE, Kallmes DF, Kashani K. Postcontrast acute kidney injury in intensive care unit patients: a propensity score-adjusted study. Intensive Care Med. 2017;43(6):1-11. https://doi. org/10.1007/s00134-017-4699-y.

32. Lee $Y$, et al. Contrast-induced acute kidney injury among patients with chronic kidney disease undergoing imaging studies: a meta-analysis. AJR Am J Roentgenol. 2019;213:728-35.

33. McDonald RJ, et al. Intravenous contrast material exposure is not an independent risk factor for dialysis or mortality. Radiology. 2014;273(3):714-25. https://doi.org/10.1148/radiol.14132418.

34. McDonald JS, McDonald RJ, Carter RE, Katzberg RW, Kallmes DF, Williamson EE. Risk of intravenous contrast material-mediated acute kidney injury: a propensity score-matched study stratified by baselineestimated glomerular filtration rate. Radiology. 2014;271(1):65-73. https://doi.org/10.1148/radiol.13130775.

35. Hinson JS, et al. Risk of acute kidney injury after intravenous contrast media administration. Ann Emerg Med. 2017;69(5):577-586.e4. https:// doi.org/10.1016/j.annemergmed.2016.11.021.

36. Aycock RD, Westafer LM, Boxen JL, Majlesi N, Schoenfeld EM, Bannuru RR. Acute kidney injury after computed tomography: a meta-analysis. Ann Emerg Med. 2018;71(1):44-53.e4. https://doi.org/10.1016/j.annem ergmed.2017.06.041.

37. Wilhelm-Leen E, Montez-Rath ME, Chertow G. Estimating the risk of radiocontrast-associated nephropathy. J Am Soc Nephrol. 2017;28(2):653-9. https://doi.org/10.1681/ASN.2016010021.

38. Barat $M$, et al. Unenhanced CT for clinical triage of elderly patients presenting to the emergency department with acute abdominal pain. Diagn Interv Imaging. 2019. https://doi.org/10.1016/j.diii.2019.05.004.

39. Thorisson A, Nikberg M, Torkzad MR, Laurell H, Smedh K, Chabok A. Diagnostic accuracy of acute diverticulitis with unenhanced low-dose CT. BJS Open. 2020;4(4):659-65. https://doi.org/10.1002/bjs5.50290.

40. Abu-Zidan FM, Cevik AA. Diagnostic point-of-care ultrasound (POCUS) for gastrointestinal pathology: state of the art from basics to advanced. World J Emerg Surg. 2018;13(1):1-14. https://doi.org/10.1186/ s13017-018-0209-y

41. Valentino M, Serra C, Ansaloni L, Mantovani G, Pavlica P, Barozzi L. Sonographic features of acute colonic diverticulitis. J Clin Ultrasound. 2009;37(8):457-63. https://doi.org/10.1002/jcu.20619.

42. Chabok A, Påhlman L, Hjern F, Haapaniemi S, Smedh K. Randomized clinical trial of antibiotics in acute uncomplicated diverticulitis. Br J Surg 2012:99(4):532-9. https://doi.org/10.1002/bjs.8688.

43. Daniels $L$, et al. Randomized clinical trial of observational versus antibiotic treatment for a first episode of CT-proven uncomplicated acute diverticulitis. Br J Surg. 2017;104(1):52-61. https://doi.org/10.1002/bjs. 10309.

44. Tandon A, Fretwell VL, Nunes QM, Rooney PS. Antibiotics vs no antibiotics in the treatment of acute uncomplicated diverticulitis - a systematic review and meta-analysis. Colorectal Dis. 2018;20(3):179-88. https://doi. org/10.1111/codi.14013.

45. Mocanu $\mathrm{V}$, et al. The role of antibiotics in acute uncomplicated diverticulitis: a systematic review and meta-analysis. Am J Surg. 2018:216(3):604-9. https://doi.org/10.1016/j.amjsurg.2018.01.039.

46. Huston JM, Zuckerbraun BS, Moore LJ, Sanders JM, Duane TM. Antibiotics versus no antibiotics for the treatment of acute uncomplicated diverticulitis: review of the evidence and future directions. Surg Infect (Larchmt). 2018;19(7):648-54. https://doi.org/10.1089/sur.2018.115.

47. Andersen JC, et al. Danish national guidelines for treatment of diverticular disease. Dan Med J. 2012;59(5):C4453.
48 Ambrosetti P, Chautems R, Soravia C, Peiris-Waser N, Terrier F. Longterm outcome of mesocolic and pelvic diverticular abscesses of the left colon: a prospective study of 73 cases. Dis Colon Rectum. 2005;48:787-91.

49. Brandt D, Gervaz P, Durmishi Y, Platon A, Morel P, Poletti PA. Percutaneous CT scan-guided drainage versus antibiotherapy alone for Hinchey II diverticulitis: a case-control study. Dis Colon Rectum. 2006;49:1533-8.

50. Kumar RR, et al. Factors affecting the successful management of intra-abdominal abscesses with antibiotics and the need for percutaneous drainage. Dis Colon Rectum. 2006. https://doi.org/10.1007/ s10350-005-0274-7.

51 Singh B, May K, Coltart I, Moore NR, Cunningham C. The long-term results of percutaneous drainage of diverticular abscess. Ann R Coll Surg Engl. 2008;90:297-301.

52. Gregersen R, Mortensen LQ, Burcharth J, Pommergaard HC, Rosenberg J. Treatment of patients with acute colonic diverticulitis complicated by abscess formation: a systematic review. Int J Surg. 2016;35(2016):201-8. https://doi.org/10.1016/j.ijsu.2016.10.006.

53 Mali J, Mentula P, Leppäniemi A, Sallinen V. Determinants of treatment and outcomes of diverticular abscesses. World J Emerg Surg. 2019;14(1):31. https://doi.org/10.1186/s13017-019-0250-5.

54. Sallinen VJ, Mentula PJ, Leppäniemi AK. Nonoperative management of perforated diverticulitis with extraluminal air is safe and effective in selected patients. Dis Colon Rectum. 2014. https://doi.org/10.1097/ DCR.0000000000000083.

55. Dharmarajan S, Hunt SR, Birnbaum EH, Fleshman JW, Mutch MG. The efficacy of nonoperative management of acute complicated diverticulitis. Dis Colon Rectum. 2011. https://doi.org/10.1007/DCR.0b013e3182 0ef759.

56 Costi R, Cauchy F, Le Bian A, Honart JF, Creuze N, Smadja C. Challenging a classic myth: pneumoperitoneum associated with acute diverticulitis is not an indication for open or laparoscopic emergency surgery In hemodynamically stable patients. A 10-year experience with a nonoperative treatment. Surg Endosc. 2012. https://doi.org/10.1007/ s00464-012-2157-z.

57. Devaraj B, Liu W, Tatum J, Cologne K, Kaiser AM. Medically treated diverticular abscess associated with high risk of recurrence and disease complications. Dis Colon Rectum. 2016;59(3):208-15. https://doi.org/ 10.1097/DCR.0000000000000533.

58. Jalouta T, Jrebi N, Luchtefeld M, Ogilvie JW. Diverticulitis recurrence after percutaneous abscess drainage. Int J Colorectal Dis. 2017:32(10):1367-73. https://doi.org/10.1007/s00384-017-2825-5.

59. Garfinkle R, et al. Diverticular abscess managed with long-term definitive nonoperative intent is safe. Dis Colon Rectum. 2016;59(7):648-55. https://doi.org/10.1097/DCR.0000000000000624.

60. Lidor AO, Schneider E, Segal J, Yu Q, Feinberg R, Wu AW. Elective surgery for diverticulitis is associated with high risk of intestinal diversion and hospital readmission in older adults. J Gastrointest Surg. 2010;14(12):1867-74. https://doi.org/10.1007/s11605-010-1344-2.

61. Hwang SS, Cannom RR, Abbas MA, Etzioni D. Diverticulitis in transplant patients and patients on chronic corticosteroid therapy: a systematic review. Dis Colon Rectum. 2010;53(12):1699-707. https://doi.org/10. 1007/DCR.0b013e3181f5643c

62 Dalla Valle $\mathrm{R}$, et al. Acute diverticulitis with colon perforation in renal transplantation. Transplant Proc. 2005;37(6):2507-10. https://doi.org/10. 1016/j.transproceed.2005.06.059.

63. Qasabian RA, Meagher AP, Lee R, Dore GJ, Keogh A. Severe diverticulitis after heart, lung, and heart-lung transplantation. J Hear Lung Transplant. 2004;23(7):845-9. https://doi.org/10.1016/j.healun.2003.07.019.

64. Biondo S, Trenti L, Elvira J, Golda T, Kreisler E. Outcomes of colonic diverticulitis according to the reason of immunosuppression. Am J Surg. 2016:212(3):384-90. https://doi.org/10.1016/j.amjsurg.2016.01.038.

65. Jin-Dominguez $F$, et al. Epidemiology of diverticulitis and prevalence of first-ever colorectal cancer postdiverticulitis in adults in the united states: a population-based national study. Dis Colon Rectum. 2021;64(2):181-9. https://doi.org/10.1097/DCR.0000000000001837.

66. Meyer J, Buchs NC, Ris F. Colonoscopy should be performed after an episode of uncomplicated diverticulitis. Dig Surg. 2019;36(4):357. https://doi.org/10.1159/000493436.

67. Meyer J, et al. Risk of colorectal cancer in patients with acute diverticulitis: a systematic review and meta-analysis of observational studies. 
Clin Gastroenterol Hepatol. 2019;17(8):1448-1456.e17. https://doi. org/10.1016/j.cgh.2018.07.031.

68. Soh NYT, Chia DKA, Teo NZ, Ong CJM, Wijaya R. Prevalence of colorectal cancer in acute uncomplicated diverticulitis and the role of the interval colonoscopy. Int J Colorectal Dis. 2018;33(7):991-4. https:// doi.org/10.1007/s00384-018-3039-1.

69. Andrade P, Ribeiro A, Ramalho R, Lopes S, MacEdo G. Routine colonoscopy after acute uncomplicated diverticulitis - challenging a putative indication. Dig Surg. 2017;34(3):197-202. https://doi.org/10. 1159/000449259.

70. Schultz JK, et al. European Society of Coloproctology: guidelines for the management of diverticular disease of the colon. Colorectal Dis. 2020;22(S2):5-28. https://doi.org/10.1111/codi.15140.

71. Schultz JK, et al. Laparoscopic lavage vs primary resection for acute perforated diverticulitis: the SCANDIV randomized clinical trial. JAMA. 2015;314(13):1364-75. https://doi.org/10.1001/jama.2015.12076.

72. Vennix S, et al. Laparoscopic peritoneal lavage or sigmoidectomy for perforated diverticulitis with purulent peritonitis: a multicentre, parallel-group, randomised, open-label trial. Lancet. 2015;386(10000):1269-77. https://doi.org/10.1016/S0140-6736(15) 61168-0.

73. Angenete $E$, et al. Laparoscopic lavage is feasible and safe for the treatment of perforated diverticulitis with purulent peritonitis. Ann Surg. 2016;263(1):117-22. https://doi.org/10.1097/SLA.0000000000 001061.

74. Ceresoli M, Coccolini F, Montori G, Catena F, Sartelli M, Ansaloni L. Laparoscopic lavage in perforated purulent diverticulitis-is it time for definitive conclusions? Int J Colorectal Dis. 2017;32(1):159. https://doi. org/10.1007/s00384-016-2674-7.

75 Ceresoli M, Coccolini F, Ansaloni L. Twelve-month results of laparoscopic lavage in perforated acute diverticulitis—update of meta-analysis results. New evidences, which perspectives? J Trauma Acute Care Surg. 2017. https://doi.org/10.1097/TA.00000000000001674.

76 Ceresoli M, Coccolini F, Montori G, Catena F, Sartelli M, Ansaloni L. Laparoscopic lavage versus resection in perforated diverticulitis with purulent peritonitis: a meta-analysis of randomized controlled trials. World J Emerg Surg. 2016;11:1-7.

77 Penna M, Markar SR, Mackenzie H, Hompes R, Cunningham C. Laparoscopic lavage versus primary resection for acute perforated diverticulitis. Ann Surg. 2017. https://doi.org/10.1097/SLA.0000000000002236.

78. Acuna SA, et al. Operative strategies for perforated diverticulitis: a systematic review and meta-analysis. Dis Colon Rectum. 2018;61(12):144253. https://doi.org/10.1097/DCR.0000000000001149.

79 Azhar N, et al. Laparoscopic lavage vs primary resection for acute perforated diverticulitis: long-term outcomes from the scandinavian diverticulitis (SCANDIV) randomized clinical trial. JAMA Surg. 2020. https://doi.org/10.1001/jamasurg.2020.5618.

80. Cirocchi R, et al. A historical review of surgery for peritonitis secondary to acute colonic diverticulitis: from Lockhart-Mummery to evidencebased medicine. World J Emerg Surg. 2017;12(1):1-24. https://doi.org/ 10.1186/s13017-017-0120-y.

81. Vermeulen J, Lange JF. Treatment of perforated diverticulitis with generalized peritonitis: past, present, and future. World I Surg. 2010;34(3):587-93. https://doi.org/10.1007/s00268-009-0372-0.

82. Halim H, Askari A, Nunn R, Hollingshead J. Primary resection anastomosis versus Hartmann's procedure in Hinchey III and IV diverticulitis. World J Emerg Surg. 2019;14(1):4-11. https://doi.org/10.1186/ s13017-019-0251-4.

83. Gachabayov M, Tuech JJ, Tulina I, Coget J, Bridoux V, Bergamaschi R. Primary anastomosis and nonrestorative resection for perforated diverticulitis with peritonitis: meta-analysis of randomized trials. Colorectal Dis. 2020;22(10):1245-57. https://doi.org/10.1111/codi.15016.

84. Goldstone RN, Cauley CE, Chang DC, Kunitake H, Ricciardi R, Bordeianou $L$. The effect of surgical training and operative approach on outcomes in acute diverticulitis: should guidelines be revised? Dis Colon Rectum. 2019:62(1):71-8. https://doi.org/10.1097/DCR.0000000000001240.

85. Sartelli $\mathrm{M}$, et al. Management of intra-abdominal infections: recommendations by the WSES 2016 consensus conference. World J Emerg Surg. 2017;12(1):1-31. https://doi.org/10.1186/s13017-017-0132-7.
86. Sohn M, et al. Perforated diverticulitis with generalized peritonitis: low stoma rate using a 'damage control strategy'.World J Surg. 2018;42(10):3189-95. https://doi.org/10.1007/s00268-018-4585-y.

87. Perathoner A, Klaus A, Mühlmann G, Oberwalder M, Margreiter R, KafkaRitsch R. Damage control with abdominal vacuum therapy (VAC) to manage perforated diverticulitis with advanced generalized peritonitisa proof of concept. Int J Colorectal Dis. 2010;25(6):767-74. https://doi. org/10.1007/s00384-010-0887-8.

88. Kafka-Ritsch $R$, et al. Damage control surgery with abdominal vacuum and delayed bowel reconstruction in patients with perforated diverticulitis Hinchey III/IV. J Gastrointest Surg. 2012;16(10):1915-22. https:// doi.org/10.1007/s11605-012-1977-4.

89. Tartaglia $\mathrm{D}$, et al. Damage control surgery for perforated diverticulitis with diffuse peritonitis: Saves lives and reduces ostomy. World J Emerg Surg. 2019;14(1):10-5. https://doi.org/10.1186/s13017-019-0238-1.

90. Cirocchi $\mathrm{R}$, et al. Role of damage control surgery in the treatment of Hinchey III and IV sigmoid diverticulitis: a tailored strategy. Medicine (United States). 2014;93(25):1-7. https://doi.org/10.1097/MD.00000 00000000184

91. Sohn M, Agha A, Heitland W, Gundling F, Steiner P, lesalnieks I. Damage control strategy for the treatment of perforated diverticulitis with generalized peritonitis. Tech Coloproctol. 2016;20(8):577-83. https://doi. org/10.1007/s10151-016-1506-7.

92. Ordoñez CA, et al. Damage control surgery may be a safe option for severe non-trauma peritonitis management: proposal of a new decision-making algorithm. World J Surg. 2020. https://doi.org/10.1007/ s00268-020-05854-y.

93. Esparza-Monzavi CA, Naffouje SA, Chaudhry V, Nordenstam J, Mellgren A, Gantt G. Open vs minimally invasive approach for emergent colectomy in perforated diverticulitis. Dis Colon Rectum. 2020. https://doi. org/10.1097/dcr.0000000000001805.

94. Hall J, et al. The American Society of Colon and Rectal Surgeons Clinical Practice Guidelines for the treatment of left-sided colonic diverticulitis. Dis Colon Rectum. 2020;6:728-47. https://doi.org/10.1097/DCR.00000 00000001679

95. Vennix S, et al. Emergency laparoscopic sigmoidectomy for perforated diverticulitis with generalised peritonitis: a systematic review. Dig Surg. 2016:33(1):1-7. https://doi.org/10.1159/000441150.

96. Vennix S, et al. Acute laparoscopic and open sigmoidectomy for perforated diverticulitis: a propensity score-matched cohort. Surg Endosc. 2016;30(9):3889-96. https://doi.org/10.1007/s00464-015-4694-8.

97. Sartelli $\mathrm{M}$, et al. The management of intra-abdominal infections from a global perspective: 2017 WSES guidelines for management of intraabdominal infections. World J Emerg Surg. 2017;12(1):1-34. https://doi. org/10.1186/s13017-017-0141-6.

98. Cicuttin $\mathrm{E}$, et al. Trends in open abdomen management in Italy: a subgroup analysis from the IROA project. Updates Surg. 2019. https:// doi.org/10.1007/s13304-019-00687-4.

99. Sawyer RG, et al. Trial of short-course anti-microbial therapy for intraabdominal infection. N Engl J Med. 2015;372(21):1996-2005. https://doi. org/10.1056/NEJMoa1411162.

100. Hanretty AM, Gallagher JC. Shortened courses of antibiotics for bacterial infections: a systematic review of randomized controlled trials. Pharmacotherapy. 2018;38(6):674-87. https://doi.org/10.1002/phar. 2118.

\section{Publisher's Note}

Springer Nature remains neutral with regard to jurisdictional claims in published maps and institutional affiliations. 